\title{
Quel montant devrais-je consacrer au marketing?
}

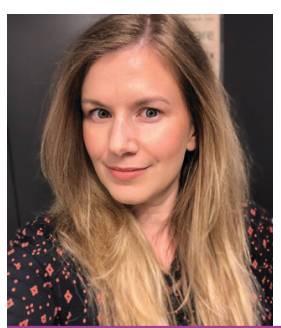

Zoey Duncan

Zoey Duncan est stratège de contenu chez Marketing4ECPs, où elle crée du contenu personnalisé imprégné de la touche particulière de chaque client. Zoey écrit et révise depuis plus d'une décennie en tant que journaliste, auteure et prête-plume. Lorsqu'elle n'est pas à son clavier, vous trouverez sûrement Zoey en train de coudre ses propres vêtements ou de se promener à vélo dans Calgary. Pour en savoir plus sur Zoey et sur son entreprise Marketing4ECPs, visitez le site www.marketing4ecps.com.

$\mathrm{L}$

e montant que vous consacrez à des fins de marketing ne constitue pas une simple dépense comme le loyer pour votre espace professionnel - c'est un investissement. Vous injectez des capitaux dans votre entreprise afin de la faire croître, d'attirer des patients et d'augmenter vos profits.

Si vos investissements en marketing dans le passé n'ont pas rapporté les résultats escomptés, il se peut que vous n'ayez pas dépensé suffisamment pour avoir un impact, que le suivi ait été difficile ou que votre campagne n'ait pas été organisée correctement (p. ex. le ciblage d'une campagne de publicité numérique). L'établissement d'un chiffre de départ idéal pour votre investissement en marketing dépendra de la croissance que vous souhaitez connaître et, surtout, de votre situation actuelle.

\section{DRESSEZ VOTRE PORTRAIT FINANCIER GLOBAL}

On vous a peut-être déjà conseillé de dépenser $5 \%$ des ventes de l'an dernier ou encore $7 \%$ des ventes prévues. Quoique ces chiffres puissent s'appliquer dans certaines industries et circonstances, ils ne sont pas universels. Un meilleur point de départ pour un cabinet de soins oculaires est d'examiner vos bénéfices des 12 derniers mois.

\section{Analysez votre parcours pour mieux cerner vos options pour l'avenir}

En admettant que la supposition suivante soit vraie, si au cours des 12 prochains mois vous déboursez le même montant sur le marketing que pour la même période l'an dernier, vous obtiendrez les mêmes résultats. Cela signifie que vous pouvez vous fonder sur les années antérieures pour établir votre budget de marketing.

Si votre cabinet a réalisé un profit de 1 million de dollars au cours des 12 derniers mois et que vous avez dépensé $25000 \$$ en marketing pour y arriver, cela représente des coûts de marketing de 2,5\%.

Si vous comptez augmenter votre bénéfice de $20 \%$ au cours de l'année prochaine pour le porter à 1,2 million de dollars, vous devrez parallèlement augmenter vos dépenses en marketing. Un bon indicateur serait d'investir 10 \% de la croissance souhaitée. Dans l'exemple susmentionné, pour obtenir une croissance de $200000 \$$, il faut ajouter $20000 \$$ au budget de marketing, portant son total à $45000 \$$.

\section{DES SUBTILITÉS NON NÉGLIGEABLES}

Mais comment faire pour répartir judicieusement cet investissement en marketing? Vous (ou votre société de commercialisation) devez absolument connaître les chiffres clés à petite échelle relatifs à chaque patient. C'est ce qui vous permet de gérer efficacement votre budget de marketing, qu'il s'agisse de créer un site Web qui convertit les visiteurs en patients ou de mener des campagnes de publicité numérique.

\section{Revenu par patient}

Connaissez-vous les coûts associés à chaque nouveau patient? Il s'agit d'un chiffre clé qu'il faut surveiller à l'interne, car celui-ci déterminera la fourchette de dépenses optimale pour chaque nouveau patient. Nous constatons que le revenu moyen généré par patient se situe habituellement entre 300 et $500 \$$. 


\section{Coût d'acquisition des patients}

Lorsque vous connaissez ce que rapporte chaque patient, vous pouvez déterminer le coût d'acquisition des patients en général. Pour reprendre notre exemple précédent, si votre patient rapporte en moyenne 500 , vous seriez probablement porté à investir $15 \%$ de ce revenu, ou 75 \$, pour acquérir un nouveau patient. Sur la plupart des feuilles de calcul, un investissement initial de 75 \$ qui vous en rapportera $500 \$$ par la suite a sans doute belle allure.

\section{Votre taux de conversion des clients potentiels}

Vous êtes bien conscient que vous ne pouvez pas vous attendre à ce que chaque personne qui visite votre site Web ou qui voit l'une de vos annonces devienne un patient. Vous devez toutefois savoir combien de vos clients potentiels deviendront des patients. Il s'agit de votre taux de conversion des clients potentiels.

Si ce n'est pas déjà une donnée que vous suivez actuellement, vous pouvez estimer que, pour un cabinet de soins oculaires, environ 30 à $50 \%$ de vos clients éventuels se convertiront en patients. Si votre cabinet a un taux de conversion des clients potentiels de $50 \%$, vous devrez attirer en moyenne deux clients éventuels pour chaque patient.

En nous appuyant sur l'investissement de 75 \$ par patient cité plus haut, vous devrez prévoir des coûts de 37,50 \$ pour chaque client potentiel.

\section{Donnez le temps à votre investissement de fructifier}

Votre coût par client potentiel de 37,50 \$ est un bon point de départ pour votre campagne de marketing. L'avantage indéniable de la publicité numérique, c'est qu'il est possible de l'ajuster « à la volée ». Par exemple, si vous vous trouvez dans un marché moins concurrentiel, vous pouvez dépenser moins par client potentiel, et vice versa.

Peu importe dans quoi vous choisissez d'investir votre budget de marketing, assurez-vous simplement de donner suffisamment de temps à votre investissement pour fructifier. Au minimum, il vous faut attendre un ou deux trimestres pour noter un effet quelconque sur votre pratique.

Évidemment, ce ne sont pas de telles données qui vous ont poussé à vous consacrer aux soins oculaires. Cependant, en aiguisant votre compréhension à la fois de votre portrait financier global et des subtilités non négligeables, vous découvrirez que vous possédez les ressources pour pousser votre pratique vers de nouveaux sommets.

Enfin, la publicité numérique bien faite devrait vous rapporter de l'argent, et non l'inverse. Elle est presque toujours rentable. 